\title{
Diffusion-weighted imaging of hepatocellular carcinoma before and after transarterial chemoembolization: role in survival prediction and response evaluation
}

\author{
Tim A. Labeur ${ }^{1,2}$ • Jurgen H. Runge ${ }^{1}$ • Elisabeth G. Klompenhouwer ${ }^{3} \cdot$ Heinz-Josef Klümpen $^{2,4} \cdot$ R. Bart Takkenberg $^{5}$. \\ Otto M. van Delden ${ }^{1}$
}

Published online: 8 May 2019

(c) The Author(s) 2019

\begin{abstract}
Background Survival outcomes of patients with hepatocellular carcinoma (HCC) treated with transarterial chemoembolization (TACE) are heterogeneous. Measuring the apparent diffusion coefficient (ADC) using diffusion-weighted imaging (DWI) may improve overall survival prediction.

Aim To assess the value of measuring the ADC before and after TACE in predicting overall survival.

Methods A retrospective analysis was performed in HCC patients treated with TACE at a tertiary referral center between 2008 and 2017. The ADC values and changes in ADC value ( $\Delta$ ADC) of HCC lesions $(\geq 1 \mathrm{~cm})$ and liver parenchyma were assessed by DWI $\leq 3$ months before and after first TACE. Pre- and post-TACE ADC values were compared with tumor response according to mRECIST and correlated with overall survival (OS) in a univariable and multivariable Cox-regression analysis.

Results A total of 89 patients were included, mostly Child-Pugh A (85\%) and BCLC stage B (53\%) with a median OS of 21.7 months (95\% CI 17.6-25.9). Tumor ADC increased from $1081 \mathrm{~mm}^{2} / \mathrm{s}$ before (IQR 964-1225) to $1328 \mathrm{~mm} / \mathrm{s}$ (IQR 1197-1560) after TACE $(p<0.001)$. Responders according to mRECIST showed a higher $\triangle A D C$ after first TACE than nonresponders (26 vs. 14\%, $p=0.048$ ). Pre-TACE ADC and $\triangle \mathrm{ADC}$ were not significantly associated with OS in both univariable and multivariable analysis, whereas response according to mRECIST remained an independent predictor of OS.

Conclusion mRECIST was confirmed as an independent prognostic factor of OS, but pre- or post-TACE ADC measurements were not. Response according to mRECIST was associated with a higher increase in ADC than non-response.
\end{abstract}

Keywords Hepatocellular carcinoma · Transarterial chemoembolization · Diffusion-weighted imaging · DWI · Apparent diffusion coefficient $\cdot$ Overall survival

Electronic supplementary material The online version of this article (https://doi.org/10.1007/s00261-019-02030-2) contains supplementary material, which is available to authorized users.

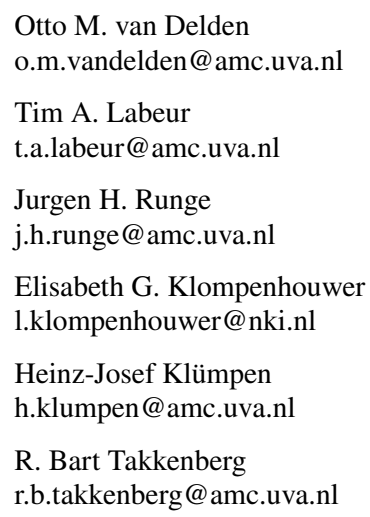

\section{Abbreviations \\ ADC Apparent diffusion coefficient \\ AFP Alpha-fetoprotein}

1 Department of Radiology and Nuclear Medicine, Amsterdam University Medical Centers, University of Amsterdam, Meibergdreef 9, 1105 AZ Amsterdam, NL, The Netherlands

2 Cancer Center Amsterdam, Amsterdam, The Netherlands

3 Department of Radiology, The Netherlands Cancer Institute, Amsterdam, The Netherlands

4 Department of Medical Oncology, Amsterdam University Medical Centers, University of Amsterdam, Meibergdreef 9, Amsterdam, The Netherlands

5 Department of Gastroenterology and Hepatology, Amsterdam University Medical Centers, University of Amsterdam, Meibergdreef 9, Amsterdam, The Netherlands 


$\begin{array}{ll}\text { AUROC } & \begin{array}{l}\text { Area under the receiver operating } \\ \text { characteristics }\end{array} \\ \text { BCLC } & \begin{array}{l}\text { Barcelona clinic liver cancer } \\ \text { Complete response }\end{array} \\ \text { CR } & \begin{array}{l}\text { Computed tomography } \\ \text { CT }\end{array} \\ \text { cTACE } & \begin{array}{l}\text { Conventional transarterial } \\ \text { chemoembolization }\end{array} \\ \text { DEB-TACE } & \begin{array}{l}\text { Drug-eluting beads transarterial } \\ \text { chemoembolization }\end{array} \\ \text { DWI } & \text { Diffusion-weighted imaging } \\ \text { EASL } & \text { European Association for the Study of the } \\ & \text { Liver } \\ \text { ECOG PS } & \begin{array}{l}\text { Eastern Cooperative Oncology Group Per- } \\ \text { formance status }\end{array} \\ \text { EPI } & \text { Echo planar imaging } \\ \text { HCC } & \text { Hepatocellular carcinoma } \\ \text { IQR } & \text { Interquartile range } \\ \text { MDT } & \text { Multidisciplinary team } \\ \text { mRECIST } & \text { Modified response evaluation criteria in } \\ & \text { solid tumors } \\ \text { MRI } & \text { Magnetic resonance imaging } \\ \text { OS } & \text { Overall survival } \\ \text { PD } & \text { Progressive disease } \\ \text { PFS } & \text { Progression-free survival } \\ \text { PR } & \text { Partial response } \\ \text { RFA } & \text { Radiofrequency ablation } \\ \text { ROI } & \text { Region of interest } \\ \text { SD } & \text { Stabile disease } \\ \text { TACE } & \text { Transarterial chemoembolization } \\ \text { TTP } & \text { Time to progression }\end{array}$

\section{Introduction}

Worldwide, transarterial chemoembolization (TACE) is the most frequently applied treatment for patients with unresectable hepatocellular carcinoma (HCC) [1]. Survival outcomes following TACE are highly variable and individual patients outcomes, ranging between 11 and 45 months, are difficult to predict $[2,3]$. It is widely acknowledged that a significant degree of variation in clinical outcomes can be attributed to differences in tumor stage (i.e., tumor size and number) and biological tumor behavior [3]. Current staging systems for HCC, including the guideline-endorsed Barcelona Clinic Liver Cancer (BCLC) staging system [2, 4], rely heavily on parameters such as performance status (Eastern Cooperative Oncology Group Performance Status (ECOG PS)), liver function (Child-Pugh score), and radiological tumor burden. The current standard for radiological response evaluation following TACE, i.e., modified Response Evaluation Criteria in Solid Tumors (mRECIST) criteria [2, 5], focusses on the change in tumoral contrast enhancement on post-TACE
CT or MRI. Improved staging and evaluation of response to TACE may be possible by more accurately assessing tumor biology. Histopathological and molecular subtyping of $\mathrm{HCC}$ is a promising technique to assess the tumor biology [6], but this requires an invasive biopsy and needs further investigation prior to implementation in clinical practice.

Diffusion-weighted imaging (DWI) is an MRI technique that detects motion of free water molecules (Brownian movement) [7]. In hypercellular tissues (i.e., fast-growing tumors), the movement of water is 'restricted' by the cellular compartments, whereas in tissue with a lower cellular density or cell necrosis, there is 'unrestricted' water diffusion. The diffusion restriction, which can be quantified into an apparent diffusion coefficient (ADC), is therefore a way of non-invasively measuring the cellularity of (tumor) tissue. Prior studies have found an inverse correlation between ADC value and histological tumor grade and microvascular invasion [8-11]. Kamel et al. first demonstrated the feasibility of DWI for response assessment after TACE by showing that an increased tumoral ADC at 4-6 weeks after TACE corresponded to radiological and histological response [12, 13]. These findings have been confirmed by several studies using DWI at various time points after TACE ( $24 \mathrm{~h}$ to 6 weeks) in association with various outcomes of TACE response (RECIST, mRECIST, histopathological necrosis, progression-free survival [PFS]) [14-19]. Less studies have described the association between pre- and post-TACE ADC value with overall survival (OS) [20-23], which remains the most robust endpoint in HCC. Moreover, the added value of DWI during routine clinical follow-up, which is often done by mRECIST at 4-8 weeks, remains unknown. Therefore, the aim of this study was to assess whether (1) pre- or postTACE ADC is a predictor of OS and if (2) ADC measurements can improve the current standard of response evaluation using mRECIST.

\section{Methods}

\section{Study population}

This retrospective study was approved by the Institutional Review Board (reference number W17_420\#17.488) which waived the need for informed consent. This study included patients with HCC treated with TACE at our center between January 2008 and December 2017 which were identified by searching the institutional patient registration system and radiology archive. Patients lacking an assessable MRI with the institutional DWI protocol ( $b$ values $50,400,800 \mathrm{~s} /$ $\left.\mathrm{mm}^{2}\right) \leq 3$ months prior to TACE or $\leq 12$ weeks after first 
TACE were excluded from the pre-TACE and post-TACE analysis, respectively.

\section{Diagnostic work-up and TACE procedure}

According to the European Association for Study of the Liver (EASL) guidelines, HCC was diagnosed by histopathological or imaging criteria [2]. All patients were discussed in a multidisciplinary team (MDT) and considered for TACE according to the Barcelona Clinic Liver Cancer (BCLC) algorithm $[2,4]$.

Accordingly, patients with intermediate stage (BCLC-B) $\mathrm{HCC}$, or those with early stage (BCLC-A) not eligible for surgery or radiofrequency ablation (RFA), were considered for TACE. Segmental portal vein invasion (BCLC-C) was not considered an absolute contra-indication for TACE.

TACE was performed using a standardized technique as described previously [24] using drug-eluting beads (DEB-TACE) loaded with doxorubicin (75-150 mg)(DC Beads 100-300 $\mu \mathrm{m}$, Terumo Europe, Belgium) or conventional TACE (cTACE) using an emulsion of doxorubicin $\left(50-75 \mathrm{mg} / \mathrm{m}^{2}\right)$ and lipiodol followed by gelatin sponge particles. Depending on size, number, and distribution of HCC lesions, uni- or bilobar, TACE was done in one or more consecutive sessions. Tumor feeders were catheterized as selectively as possible with a microcatheter, followed by chemoembolization until intra-arterial stasis was observed. If it was technically not feasible to achieve complete devascularisation in one session, an additional TACE procedure was scheduled. First follow-up was done 6 weeks after the first TACE including clinical assessment, biochemistry, and multiphasic MRI. The radiological response was assessed by expert abdominal radiologists using the mRECIST criteria [25]. Patients were reevaluated in the MDT after each follow-up visit and additional TACE was done 'on demand,' followed by clinical and radiological response assessment every 3 months.

\section{MRI technique and ADC measurement}

Pre- and post-TACE MRI were performed on a $1.5 \mathrm{~T}$ system (Siemens Healthcare, Erlangen, Germany) with combined body and spine-phased array coils. The scan protocol consisted of a T1W (axial gradient echo in- and out-of-phase) sequence, an axial and coronal T2W (fatsaturated) sequence, and axial T2W (long and short echo time) sequences followed by an axial 2D echo planar imaging (EPI) DWI sequence with $3 b$ values $(50,400,800 \mathrm{~s} /$ $\mathrm{mm}^{2}$ ) before contrast series (Fig. 1). Details of the DWI sequence are provided in Supplementary Table 1.

ADC measurements were retrospectively conducted by a trained investigator (TL), supervised by an expert abdominal radiologist (OVD, with 20 years of experience) on a high-resolution PACS workstation. The ADC value of a maximum of $3 \mathrm{HCC}$ lesions, $\geq 10 \mathrm{~mm}$ in diameter, was measured by delineating whole-lesion region of interests (ROI) at the level of the maximum transverse tumor diameter in the arterial phase. From the ADC measurements of all target lesions, the mean ADC was calculated. As a reference measurement, the ADC value of non-tumor liver parenchyma was measured by drawing a circular ROI $(\varnothing 2 \mathrm{~cm})$ on the contralateral liver lobe, while avoiding large vessels and biliary structures.

ADC values of HCC lesions and non-tumor liver parenchyma were measured on pre-TACE and post-TACE MRI. Increase in ADC value, $\triangle \mathrm{ADC}(\%)$, was defined as $\Delta \mathrm{ADC}=\left(\left(\mathrm{ADC}_{\text {post }}-\mathrm{ADC}_{\mathrm{pre}}\right) / \mathrm{ADC}_{\text {pre }}\right) \times 100$.

Several cut-offs for $\triangle \mathrm{ADC}$ have been reported in the literature $[12,15,19,20,22,23]$, therefore we analyzed 2 frequently used cut-points (13.6\% and $25 \%$ ).

Because TACE is often performed repeatedly, the ADC values at time of best mRECIST response were also measured. Best response was assessed on a patient basis, selecting the first radiological evaluation following TACE that observed the best mRECIST response category (Complete response $[\mathrm{CR}]>$ Partial response $[\mathrm{PR}]>$ Stable disease $[\mathrm{SD}]>$ Progressive disease [PD]) for that patient.

\section{Statistical analysis}

Categorical data were described as frequencies with percentages and continuous data as medians with interquartile ranges (IQR). The change in ADC value of $\mathrm{HCC}$ lesions and non-tumor liver parenchyma was assessed using the paired Wilcoxon test.

The primary endpoint, overall survival (OS), was defined from date of first TACE to date of death or last known date to be alive. Survival status was verified using the municipal records database on 4th of May 2018. If TACE was used as bridging treatment to liver resection or transplantation, OS was censored on date of surgery. Survival curves were plotted and compared using the Kaplan-Meier method and log-rank test. The association between OS and pre-TACE ADC, $\triangle \mathrm{ADC}$ and know prognostic factors [3] was assessed in a univariable Cox proportional hazard analysis. The subsequent multivariable analyses included factors that were associated with survival in univariable analysis $(p<0.1)$ and pre-TACE ADC (pre-TACE model) or $\triangle$ ADC (post-TACE model). As there are no validated cut-offs for pre-TACE ADC, we dichotomized the population according to high (above median) or low (equal to or below median) pre-TACE ADC subgroups. Additionally, the correlation between preTACE ADC and OS was expressed using the Spearman's rank correlation coefficient $(\rho)$.

To assess the association between ADC values and radiological response, patients were categorized into two 
Fig. 1 Pre- and Post-TACE contrast-enhanced (CE) MRI of a 75-year-old man with cirrhosis due to hemochromatosis and HCC. Pre-TACE MRI shows a $3.9 \mathrm{~cm}$ HCC nodule (arrows) in segment 7 of the liver with arterial enhancement (a) and delayed phase washout (c) on T1-weighted imaging. The lesion shows diffusion restriction on the Diffusion Weighted Imaging (DWI) using b-values of 50, 400, $800 \mathrm{~s} /$ $\mathrm{mm}^{2}$ (e). Post-TACE imaging shows a complete response of the HCC to TACE with complete necrosis $(\mathbf{b}, \mathbf{d})$. Only a peripheral enhancing rim, representing inflammatory change remains visible. The lesion has an increased signal on DWI (f) using the same b-values caused by T2 shine-through. The tumor ADC increased from $959 \mathrm{~mm}^{2} / \mathrm{s}$ (g) to $1564 \mathrm{~mm}^{2} / \mathrm{s} \mathrm{(h),} \mathrm{reflecting}$ a $\triangle \mathrm{ADC}$ of $63 \%$

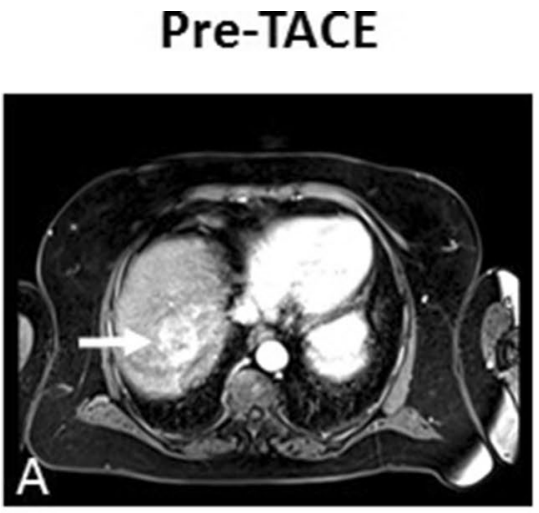

Post-TACE

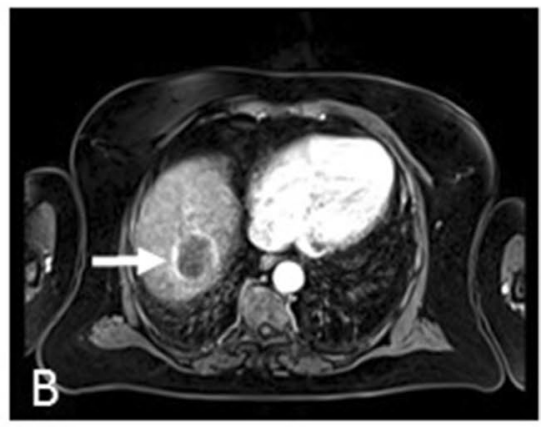

CE-T1W Arterial phase
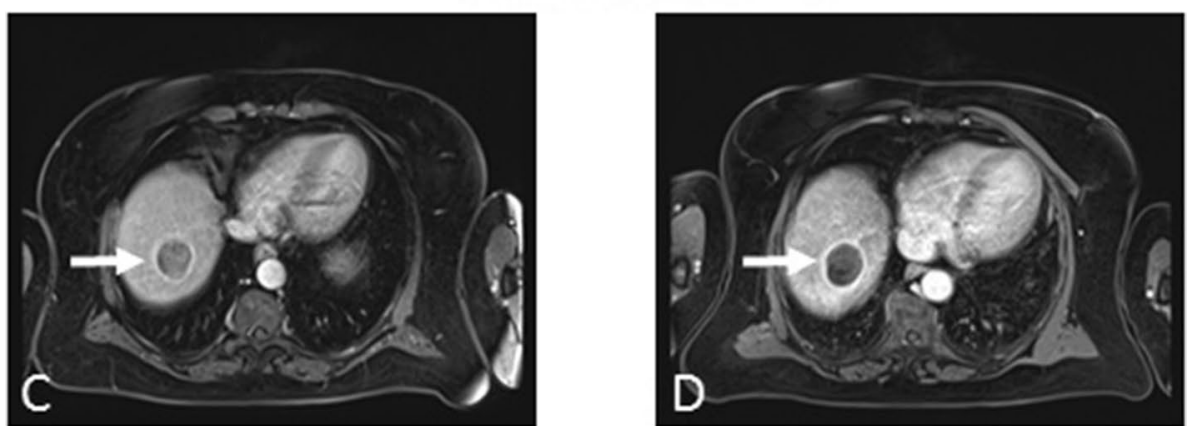

\section{CE-T1W Delayed phase}
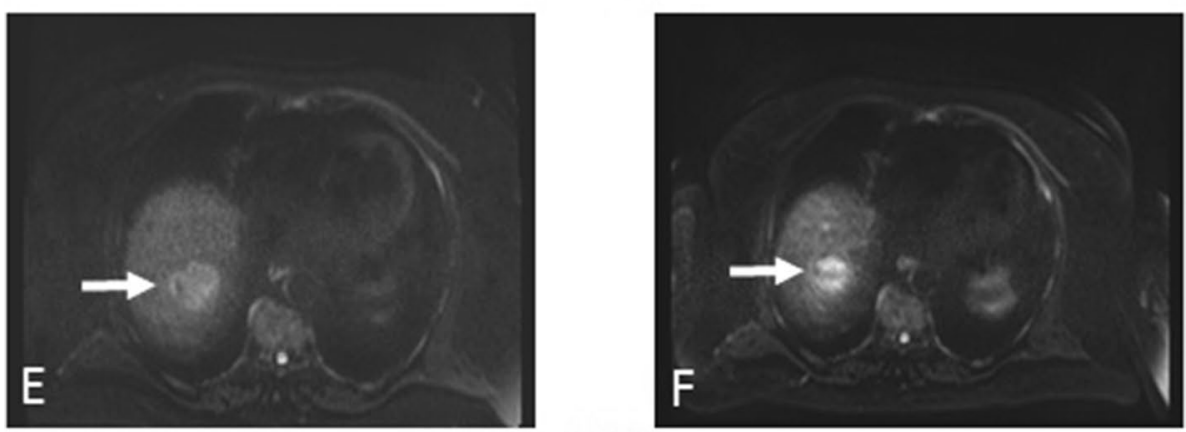

DWI

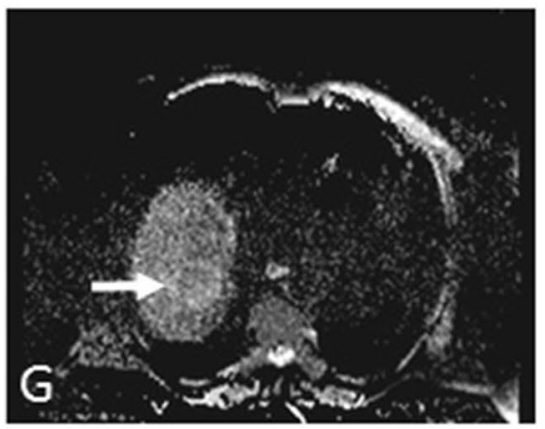

ADC

subgroups according to the mRECIST response at first follow-up. Patients with partial or complete response (PR/CR) were classified as responder, whereas non-responders had stable or progression disease (SD/PD). The Mann-Whitney test was used to compare pre- and post-TACE ADC and $\triangle \mathrm{ADC}$ between responders and non-responders. The 
area under the receiver operating characteristics (AUROC) curve was used to assess the accuracy index of $\triangle \mathrm{ADC}$ in predicting $m R E C I S T$ response and quantify the sensitivity and specificity of $\triangle \mathrm{ADC}$ cut-points.

For all statistical tests, a two-tailed $p$ value of $<0.05$ was considered statistically significant. All analyses were performed using IBM SPSS Statistics for Windows Version 24.0 (IBM Corp., Armonk, NY, USA).

\section{Results}

\section{Patients characteristics}

Of the 203 consecutive HCC patients treated with TACE between January 2008 and December 2017, 113 patients were excluded due to non-available (i.e., CT-imaging) or poor quality pre-TACE MRI-images $(n=105)$ or different $\mathrm{b}$-values of the DWI sequence $(n=8)$ (Fig. 2$)$. One patient was not assessable because of extensive tumor necrosis after embolization of tumor bleeding prior to first TACE. Consequently, 89 patients treated with TACE between September 2008 and November 2017 formed the pre-TACE study cohort. Patient characteristics and treatment details are summarized in Table 1 . The majority of patients had 1-3 tumors (75\%) with a median largest tumor size of $42 \mathrm{~mm}$ (IQR 35-58). The median number of TACE procedures was 2 (range 1-7). Fourteen patients were bridged to surgical resection or liver transplantation. At first follow-up following TACE, 52 (58\%) patients were assessed by MRI including DWI sequence and were available for post-TACE ADC analysis.

\section{Pre- and post-TACE ADC values}

The MRI was conducted a median of 5 weeks (IQR 4-8) before and 6 weeks (IQR 5-7) after first TACE, respectively. The ADC characteristics prior to and after TACE are shown in Table 2 and Fig. 3. The pre-TACE ADC measurement was not possible in $5 / 89(6 \%)$ patients or $41 / 178(23 \%)$ of the target lesions because these lesions were not visible using DWI $(12 \%)$ or too small $(<1 \mathrm{~cm})$ for a reliable measurement (11\%). At first follow-up, HCC lesions were not visible in $4 / 52(8 \%)$ patients or $18 / 98(18 \%)$ target lesions, whereas $8 / 98$ (8\%) target lesions were too small for ADC measurement. Consequently, adequate ADC measurements were possible in 85 patients with 137 target lesions pre-TACE, and 48 patients with 72 target lesions post-TACE.

The median pre-TACE ADC was $1075 \mathrm{~mm}^{2} / \mathrm{s}$ (IQR 944-1187). In the HCC lesions with both pre- and postTACE imaging, there was a significant increase between pre-TACE ADC value $\left(1081 \mathrm{~mm}^{2} / \mathrm{s}\right.$, IQR $\left.964-1225\right)$ and post-TACE ADC value $\left(1328 \mathrm{~mm}^{2} / \mathrm{s}\right.$, IQR $\left.1197-1560\right)$ $(p<0.001)$, reflecting a median increase of $22.4 \%$ (IQR 11.3-38.0\%). Non-tumor liver parenchyma did not show a significant increase in ADC value (965 to $1001 \mathrm{~mm}^{2} / \mathrm{s}$, $p=0.181)$. According to the mRECIST criteria, there was CR in 10 (19\%), PR in 25 (48\%), SD in 4 (8\%), and PD in 13 patients (25\%). Responders (CR/PR) showed a higher ADC value (1375 vs. $\left.1198 \mathrm{~mm}^{2} / \mathrm{s}, p=0.019\right)$ and $\Delta \mathrm{ADC}(25.7$ vs. $14.4 \%, p=0.048)$ after first TACE than non-responders (SD/PD). $\triangle \mathrm{ADC}$ could moderately discriminate mRECIST

Fig. 2 Consort flow diagram

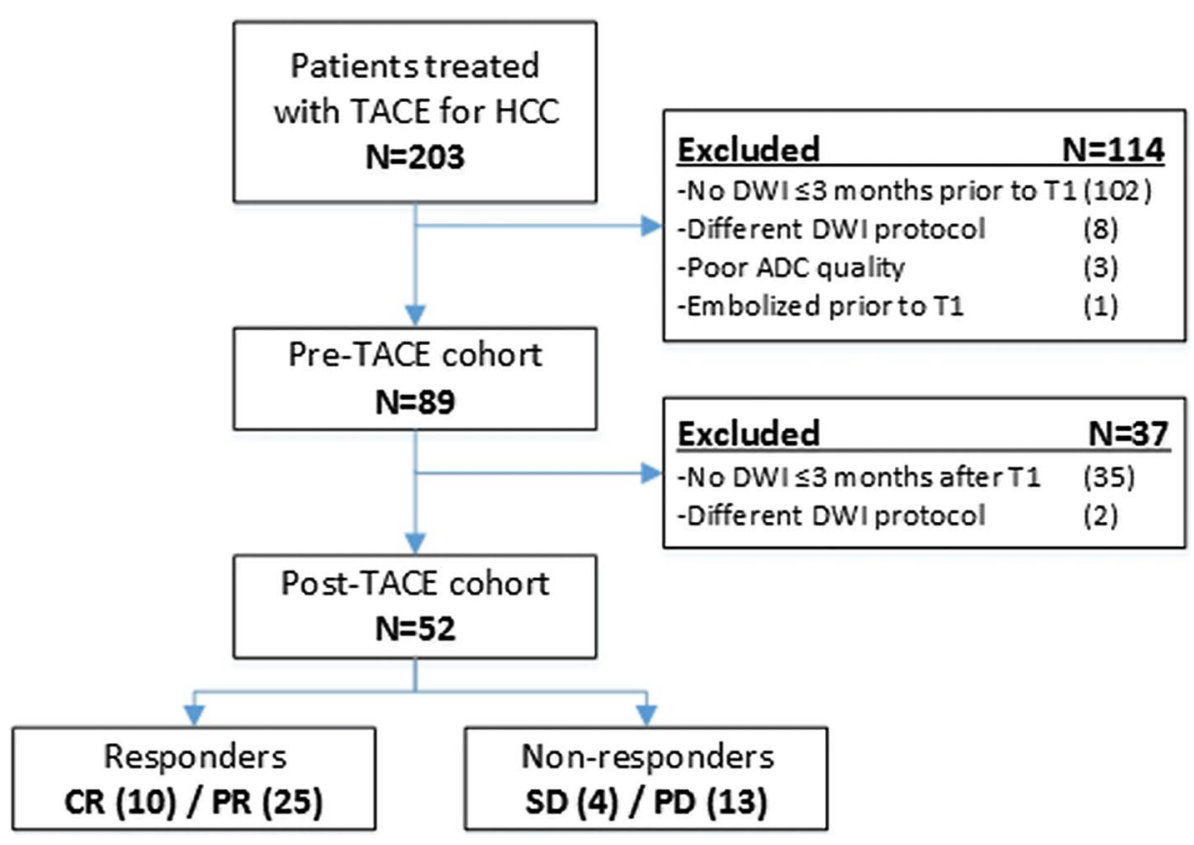


Table 1 Baseline characteristics of patients

\begin{tabular}{|c|c|}
\hline Characteristic & All patients $(N=89)$ \\
\hline \multicolumn{2}{|l|}{ Clinical parameters } \\
\hline Age-year (IQR) & $69(61-73)$ \\
\hline Males-n $(\%)$ & $70(79)$ \\
\hline \multicolumn{2}{|l|}{ Etiology- $n(\%)$} \\
\hline Alcohol & $33(37)$ \\
\hline HBV & $11(12)$ \\
\hline $\mathrm{HCV}$ & $28(32)$ \\
\hline NAFLD/NASH & $11(12)$ \\
\hline Other/unknown & $16(18)$ \\
\hline Cirrhosis- $n(\%)$ & $78(88)$ \\
\hline \multicolumn{2}{|l|}{ Child-Pugh class— $n(\%)$} \\
\hline $\mathrm{A} / \mathrm{B}$ & $76 / 13(85 / 15)$ \\
\hline \multicolumn{2}{|l|}{ ECOG PS- $n(\%)$} \\
\hline $0 / 1 / 2$ & $53 / 30 / 6(60 / 34 / 7)$ \\
\hline \multicolumn{2}{|l|}{ BCLC stage $-n(\%)$} \\
\hline 0 or $\mathrm{A} / \mathrm{B} / \mathrm{C}$ & $39 / 47 / 3(44 / 53 / 3)$ \\
\hline \multicolumn{2}{|l|}{ Number of nodes- $n(\%)$} \\
\hline $1 / 2$ or $3 />3$ & $31 / 36 / 22(35 / 41 / 25)$ \\
\hline Size of largest node-mm (IQR) & $42(35-58)$ \\
\hline Macroscopic vascular invasion $-n(\%)$ & $3(3)$ \\
\hline \multicolumn{2}{|l|}{ Treatment details } \\
\hline \multicolumn{2}{|l|}{ TACE usage- $-(\%)$} \\
\hline Bridge to liver transplantation/resection & $14(16)$ \\
\hline Received prior $\mathrm{HCC}$ treatment & $16(18)$ \\
\hline TACE was primary HCC treatment & $59(66)$ \\
\hline \multicolumn{2}{|l|}{ TACE details } \\
\hline cTACE/deb-TACE-no (\%) & $6 / 83(7 / 93)$ \\
\hline Uni-/Bilobar-no $(\%)$ & $65 / 24(73 / 27)$ \\
\hline Number of TACE (range) & $2(1-7)$ \\
\hline
\end{tabular}

$B C L C$ Barcelona Clinic Liver Cancer classification, ECOG PS Eastern Cooperative Oncology Group performance status, $H B V$ hepatitis $\mathrm{B}$ virus, $H C V$ Hepatitis $\mathrm{C}$ virus, NAFLD/NASH Non-alcoholic fatty liver disease/Non-alcoholic steatohepatitis, TACE transarterial chemoembolization

responders and non-responders with an AUROC of 0.68 (95\% CI 0.53-0.83) (Supplementary Fig. 1). For example, at the $25 \%$ cut-off, $\triangle \mathrm{ADC}$ had $52 \%$ sensitivity and $81 \%$ specificity for predicting mRECIST response, whereas this was $71 \%$ sensitivity and $50 \%$ specificity at the $13.6 \%$ cut-off for $\triangle \mathrm{ADC}$.

\section{ADC at best response according to $\mathrm{mRECIST}$}

Using repeated TACE, eight patients (15\%) achieved response after initial non-response, including $\mathrm{PD} \rightarrow \mathrm{CR}$ $(n=4), \mathrm{PD} \rightarrow \mathrm{PR}(n=2)$, and $\mathrm{SD} \rightarrow \mathrm{CR}(n=2)$. Consequently, the best response according to mRECIST was CR in $29(56 \%), \mathrm{PR}$ in $14(27 \%), \mathrm{SD}$ in $3(6 \%)$, and PD in 6 (12\%) patients. The best mRECIST response was observed after 1, 2, and 3 TACE procedures in 28 (54\%), 19 (37\%), and $5(10 \%)$ patients, respectively. MRI was conducted after a median of 10 weeks (IQR 6-22 weeks) following first TACE. Comparing with first and best response, there was a lower, albeit still significant $(p<0.001)$, increase from pre-TACE ADC value (1081 mm²/s, IQR 964-1225) to best-response ADC value (1278 mm²/s, IQR 1155-1564), reflecting a median increase of $15.7 \%$ (IQR 5.7-43.6\%). When patients were stratified according to best mRECIST response, there was no significant difference in $\triangle \mathrm{ADC}$ between non-responders and responders (11.5 vs. $16.5 \%$, $p=0.433)$.

\section{$A D C$ value and overall survival}

At the time of final survival follow-up, after a median follow-up period of 29.8 months ( $95 \%$ CI 17.3-42.3), 53 of 89 (60\%) patients had died. The median OS was 21.7 months (95\% CI 17.6-25.9). There was no significant correlation between OS and pre-TACE ADC value $(\rho=0.085, p=0.443)$ (Fig. 4a). When stratifying patients according to high $\left(>1075 \mathrm{~mm}^{2} / \mathrm{s}\right)$ or low $\left(\leq 1075 \mathrm{~mm}^{2} / \mathrm{s}\right)$ pre-TACE ADC, there were no significant differences in OS (23.8 months versus 21.7 months, $p=0.111$ ) (Fig. $4 \mathrm{~b}$ ). When included in the multivariable analysis together with known pre-TACE predictors of OS, only tumor number and serum alpha-fetoprotein (AFP) remained independent predictors (Table 3).

At first follow-up, there were significant differences in OS between responders and non-responders according to mRECIST criteria ( 24.5 vs. 18.3 months, log-rank $p=0.038$ ) (Fig. 4c). There were no significant differences in OS between patients with low or high $\triangle \mathrm{ADC}$ after first TACE, both at the $13.6 \%$ (HR 1.06, 95\% CI 0.49-2.30) and $25 \%$ cut-off (HR $0.71,95 \%$ CI $0.32-1.58$ ) (Table 3, Fig. 4d). In a second multivariable model including baseline predictors, $\triangle \mathrm{ADC}(>25 \%$ vs. $\leq 25 \%)$ and mRECIST response at first follow-up, response according to mRECIST remained independently associated with improved OS (HR 0.24, 95\% $0.08-0.74)$, whereas $\Delta$ ADC was not (HR $1.60,95 \%$ CI $0.60-4.25)$. A model including the $13.6 \%$ cut-off for $\Delta$ ADC yielded similar results (data not shown).

\section{Discussion}

In our study of HCC patients treated with TACE, we confirmed that patients with radiological tumor response as assessed per mRECIST criteria have an increased ADC after TACE. However, neither pre-TACE ADC nor $\triangle$ ADC at the routine response evaluation after first TACE were significantly associated with OS, whereas response based on the mRECIST criteria was confirmed as a significant prognostic factor. 
Table 2 ADC characteristics at first and best response

\begin{tabular}{|c|c|c|c|c|}
\hline Characteristic & All patients & Non-responders & Responders & $p$ value \\
\hline Pre-TACE & $N=89$ & & & \\
\hline ADC HCC lesion(s)—(IQR)* & 1075 (944-1187) & NA & NA & \\
\hline ADC non-tumoral liver tissue-(IQR) & $965(941-1076)$ & NA & NA & \\
\hline At first response evaluation (1FU) post-TACE & $N=52$ & $N=17$ & $N=35$ & \\
\hline $\mathrm{ADC} \mathrm{HCC}$ lesion(s) at baseline-(IQR)* & $1081(964-1225)$ & $1079(998-1218)$ & $1084(959-1230)$ & 0.956 \\
\hline $\mathrm{ADC} \mathrm{HCC}$ lesion(s) at $1 \mathrm{FU}-(\mathrm{IQR})^{* *}$ & $1328(1197-1560)$ & $1198(1125-1374)$ & $1375(1280-1574)$ & 0.019 \\
\hline ADC non-tumoral liver tissue-(IQR) & $1001(91-1077)$ & $981(853-1020)$ & $1018(914-1083)$ & 0.252 \\
\hline$\Delta \mathrm{ADC}$ HCC lesion(s) —\% (IQR)*** & $22.4(11.3-38.0)$ & $14.4(2.7-24.7)$ & $25.7(11.5-51.4)$ & 0.048 \\
\hline $\begin{array}{l}\text { At best response evaluation (BR) according to } \\
\text { mRECIST }\end{array}$ & $N=52$ & $N=9$ & $N=43$ & \\
\hline ADC HCC lesion(s) at baseline-(IQR)* & $1081(964-1225)$ & $1174(1076-1233)$ & $1081(959-1228)$ & 0.420 \\
\hline $\mathrm{ADC} \mathrm{HCC}$ lesion(s) at $\mathrm{BR}-(\mathrm{IQR}) * * * *$ & $1278(1155-1564)$ & $1198(1136-1580)$ & $1280(1174-1552)$ & 0.756 \\
\hline ADC non-tumoral liver tissue-(IQR) & $1011(892-1075)$ & $1021(838-1056)$ & 1004 (899-1079) & 0.818 \\
\hline$\Delta \mathrm{ADC}$ HCC lesion(s)—\% (IQR) ${ }^{* * * * *}$ & $15.7(5.7-43.6)$ & $11.5(1.2-28.3)$ & $16.5(5.7-49.1)$ & 0.433 \\
\hline
\end{tabular}

*Lesion(s) not visible and too small $(<1 \mathrm{~cm})$ in four and one patient, respectively

$* *$ Lesion(s) not visible in four patients

***Unable to calculate in five patients due to unmeasurable lesions pre- or post-TACE

$* * * *$ Lesion(s) not visible in five patients

*****Unable to calculate in five patients due to unmeasurable lesions pre- or post-TACE

Fig. 3 Box plots distributions of pre- and post-TACE ADC values in a tumor lesions, $\mathbf{b}$ liver parenchyma and $\mathbf{c}$ tumor lesions, stratified according to mRECIST response after first TACE. d Box plot distribution of difference in ADC $(\triangle \mathrm{ADC})$ according to mRECIST response after TACE. Boxes represent 25-75 percentiles of data values. Line in box represents median value
A

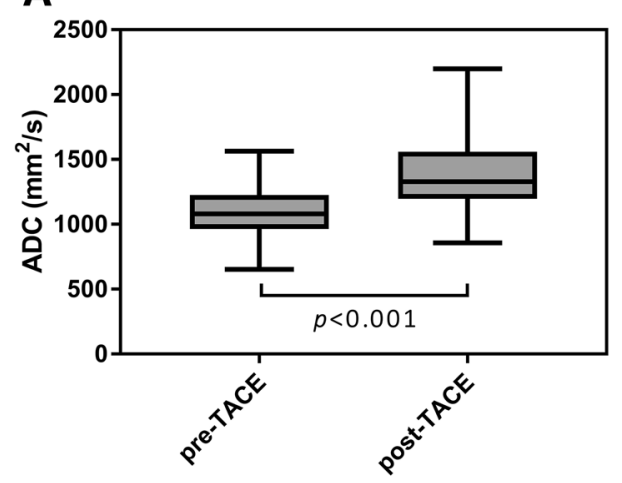

C

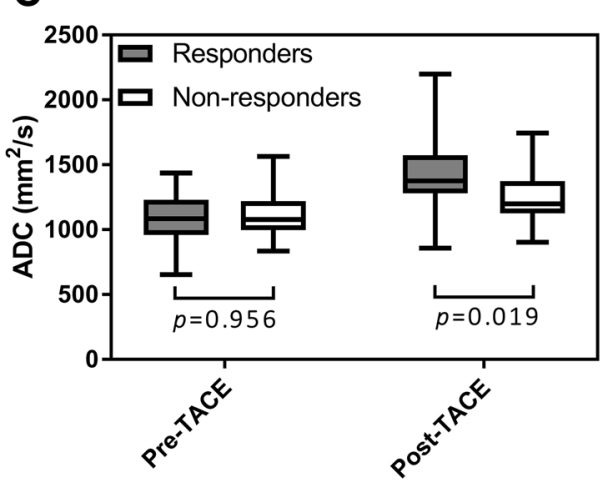

B

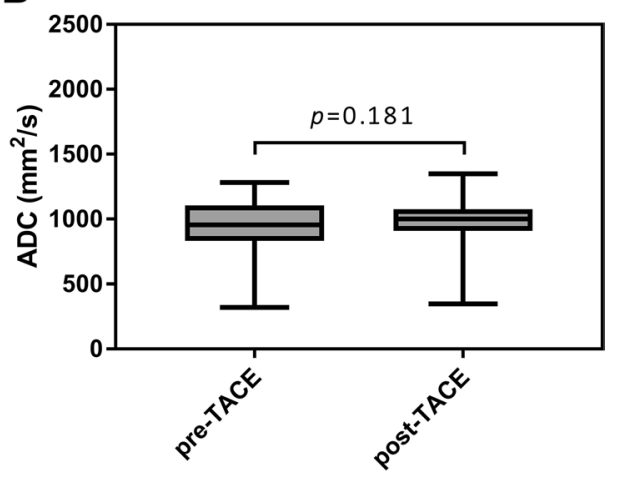

D

Tumor lesions

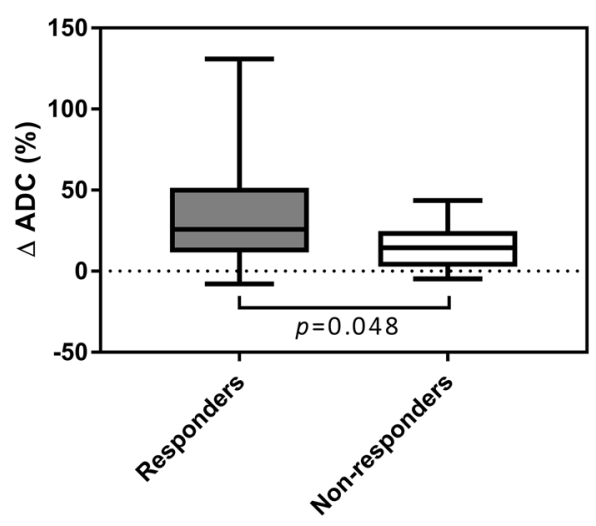


Fig. 4 The association between pre- and post-ADC measurements and overall survival. a Correlation between pre-TACE ADC and overall survival. b Overall survival according to according to high ( $>1075$ $\left.\mathrm{mm}^{2} / \mathrm{s}\right)$ or low $\left(\leq 1075 \mathrm{~mm}^{2} / \mathrm{s}\right)$ ADC prior to first TACE. c Overall survival according to mRECIST response; Complete or partial response versus stable or progressive disease. $\mathbf{d}$ Overall survival according to ADC response; High ( $>25 \%$ ) or low $(\leq 25 \%) \Delta \mathrm{ADC}$
A Correlation pre-TACE ADC \& survival
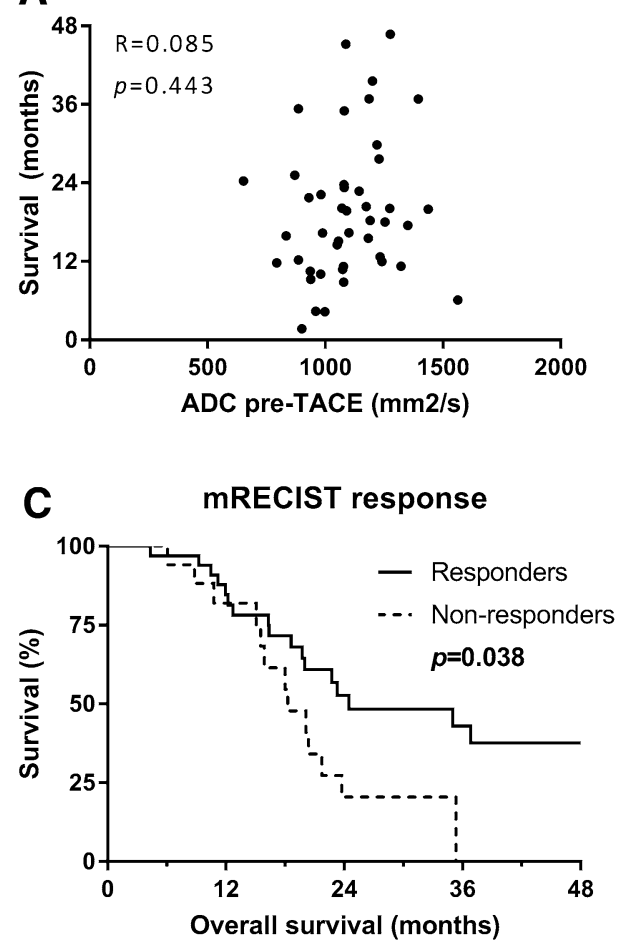

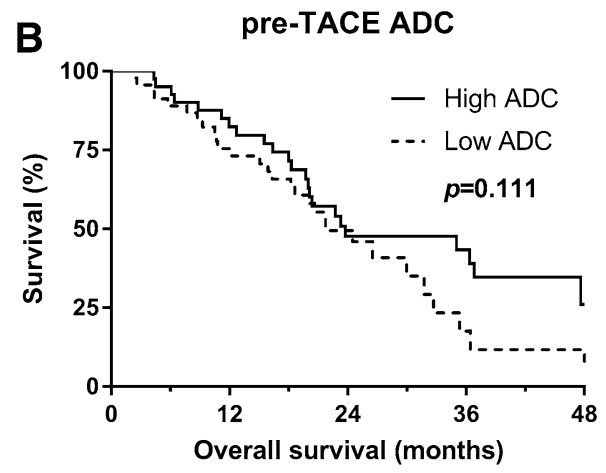

D

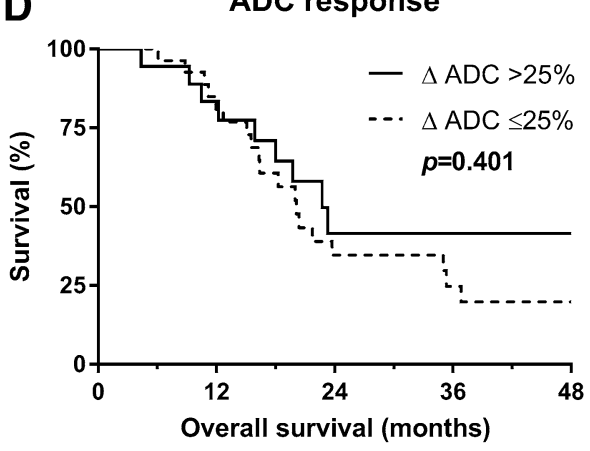

Table 3 Univariable and multivariable Cox-regression analysis for overall survival

\begin{tabular}{|c|c|c|c|c|c|c|}
\hline \multirow[t]{2}{*}{ Variables } & \multicolumn{2}{|l|}{ Univariable } & \multicolumn{2}{|l|}{$\begin{array}{l}\text { Pre-TACE }(n=89) \\
\text { Multivariable }\end{array}$} & \multicolumn{2}{|l|}{$\begin{array}{l}\text { Post-TACE }(n=52) \\
\text { Multivariable }\end{array}$} \\
\hline & HR [CI 95\%] & $p$ value $*$ & HR [CI 95\%] & $p$ value ${ }^{* *}$ & HR [CI 95\%] & $p$ value $* *$ \\
\hline \multicolumn{7}{|l|}{ Pre-TACE } \\
\hline Female sex & $0.72(0.38-1.37)$ & 0.319 & & & & \\
\hline Age $>69$ years & $1.04(0.59-1.81)$ & 0.901 & & & & \\
\hline $\mathrm{HBV}$ & $0.96(0.41-2.24)$ & 0.922 & & & & \\
\hline $\mathrm{HCV}$ & $0.95(0.54-1.69)$ & 0.870 & & & & \\
\hline Alcohol & $0.67(0.38-1.21)$ & 0.184 & & & & \\
\hline ECOG PS 2 (Ref: 0-1) & $2.17(0.92-5.11)$ & 0.076 & $2.55(0.94-6.90)$ & 0.065 & $6.97(1.56-31.07)$ & 0.011 \\
\hline Child-Pugh score B (Ref: A) & $1.48(0.75-2.94)$ & 0.262 & & & & \\
\hline Number of nodes (Ref: 1) & Ref & - & Ref & - & Ref & - \\
\hline $2-3$ & $1.66(0.87-3.18)$ & 0.125 & $1.47(0.67-3.22)$ & 0.342 & $1.14(0.40-3.23)$ & 0.812 \\
\hline$>3 /$ diffuse & $2.58(1.26-5.30)$ & 0.010 & $3.19(1.41-7.25)$ & 0.005 & $1.75(0.60-5.10)$ & 0.309 \\
\hline Tumor size $>42 \mathrm{~mm}$ & $1.81(1.04-3.15)$ & $\mathbf{0 . 0 3 7}$ & $1.61(0.88-2.96)$ & 0.125 & $2.62(0.96-7.17)$ & 0.060 \\
\hline Macrovascular invasion & $1.53(0.37-6.32)$ & 0.560 & & & & \\
\hline $\log _{10}$ AFP & $1.35(1.04-1.74)$ & 0.022 & $1.41(1.08-1.84)$ & 0.013 & $0.99(0.64-1.51)$ & 0.944 \\
\hline ADC value $>1075 \mathrm{~mm}^{2} / \mathrm{s}$ & $0.64(0.36-1.12)$ & 0.114 & $0.58(0.32-1.04)$ & 0.067 & - & - \\
\hline \multicolumn{7}{|l|}{ At first follow-up post-TACE } \\
\hline $\begin{array}{l}\text { mRECIST response (CR/PR, ref: } \\
\text { SD/PD) }\end{array}$ & $0.47(0.22-0.98)$ & 0.043 & - & - & $0.24(0.08-0.74)$ & 0.013 \\
\hline$\Delta \mathrm{ADC}>13.6 \% * * *$ & $1.06(0.49-2.30)$ & 0.875 & & & - & - \\
\hline$\Delta \mathrm{ADC}>25 \%$ & $0.71(0.32-1.58)$ & 0.404 & & & $1.60(0.60-4.25)$ & 0.350 \\
\hline
\end{tabular}

$* p$ value $<0.1$ highlighted in bold

$* * p$ value $<0.05$ highlighted in bold

***Not included in multivariable model (Post-TACE) due to collinearity with $\triangle \mathrm{ADC}>25 \%$ 
In concordance with prior studies there was a significantly higher $\triangle \mathrm{ADC}$ in patients who responded to TACE according to the mRECIST criteria $[12,14,15,19,22]$. Prior studies mainly showed the superiority of evaluating the post-TACE tumor ADC value and contrast enhancement (mRECIST) over the tumor size alone (RECIST) [12, 14, 15] or underscored the significant association between increased ADC and prolonged progression-free survival (PFS) [15, 16]. However, endpoints like PFS or time to progression (TTP) have limited accuracy in representing TACE benefit in terms of OS [2]. Overall survival remains the most robust endpoint for TACE benefit, but this requires a long follow-up (>24 months). Given the increasing number of subsequent liver-directed and systemic treatment options after TACE failure, there is an increasing clinical need for validated methods to predict OS benefit following TACE.

In our study, both pre-TACE ADC and $\triangle \mathrm{ADC}$ after first TACE were not significantly associated with OS, whereas mRECIST was an independent predictor of OS. This confirms prior studies showing the prognostic value of mRECIST response after TACE [21, 26-29]. Despite a multitude of studies on ADC measurements in HCC patients treated with TACE [8-12, 14, 15, 17-23], few other studies reported on the association between ADC and OS [20-23]. One Chinese study with limited sample size $(n=23)$ found a significant association between pre-TACE ADC and OS [23], and 4 studies showed a significant association between $\triangle \mathrm{ADC}$ and OS [20-23]. The differences in outcomes with the present study should be considered in light of the differences in study methodology. Three prior studies only reported a univariable association between $\triangle \mathrm{ADC}$ and $\mathrm{OS}[20,21,23]$, as opposed to the study by Corona et al. [22] and the present study in which more robust multivariable analyses were performed. Moreover, the timing of follow-up imaging after TACE was earlier in prior studies ( $3 \mathrm{~h}$ to 4 weeks) than our study (6 weeks). Kamel et al. described the serial changes of ADC value following TACE, showing that the largest difference in ADC value was at 1-2 weeks which declined after 3-4 weeks [30]. Serial measurements showed that the change in arterial and venous enhancement remained stable during 1-4 weeks after TACE. Consequently, the response evaluation performed in this study may have detected these (stable) differences in contrast enhancement (mRECIST), but the decreased differences in ADC value at 6 weeks could have diluted the association with OS. This was underscored by our ADC measurements at the best response to TACE (median of 10 weeks), showing a lower median ADC value than measurement after first TACE.

As ADC quantification prior to TACE and after 6 weeks did not provide better survival prediction than mRECIST in this study, more studies are needed to validate the value of earlier DWI (i.e., $<4$ weeks or $<24 \mathrm{~h}$ ) for this purpose. DWI is a short MRI sequence which does not require contrast injection, making it a promising technique for early response evaluation or in patients who are unable to receive contrast (i.e., renal failure). Replacing mRECIST at regular followup intervals appears not yet feasible due to the moderate accuracy (AUROC 0.68 ) of $\triangle \mathrm{ADC}$ in predicting treatment response according to mRECIST. Improved DWI quality or earlier response evaluation using DWI might improve the prognostic performance of DWI and ADC measurements. However, some limitations to DWI and ADC quantification should be noted. Reflected by the significant number of not-evaluable patients (6\%) and target lesions (23\%), ADC measurements are limited by inconsistent image quality and decreasing accuracy in measuring small lesions $(<1 \mathrm{~cm})$. Moreover, it is currently poorly defined how to handle the significant intra-patient (i.e., mixed tumor response) and intra-tumoral (i.e., viable and necrotic parts of lesions) heterogeneity in response evaluation using ADC. This may cause inter-observer variability and reduce the reproducibility of ADC values especially in diffuse infiltrating or partially responding tumors. According to RECIST and mRECIST criteria, mixed response is accounted for by measuring the sums of (arterial enhancing) tumor diameters [5]. Similarly, in this study we assessed TACE response on a patient basis by selecting the largest tumor diameter of a maximum of three target lesions and calculating the average ADC before and after TACE. Lastly, standardized DWI settings (i.e., $b$ values) or validated cut-offs for pre-ADC or $\triangle \mathrm{ADC}$ values are lacking. Similar to prior retrospective DWI studies [14], a large number of patients had to be excluded from this study due to differences in imaging modality $(n=102)$ or differences in DWI protocol $(n=8)$. Future prospective studies with standardized protocols are needed to address these issues prior to implementation in clinical practice.

In conclusion, in HCC patients treated with TACE, we confirmed the significant increase in ADC value in patients responding according to the mRECIST criteria. mRECIST response was independently associated with improved OS, but pre-TACE ADC and change in ADC at first routine follow-up were not significantly associated with OS. Therefore, HCC staging or response assessment at routine clinical follow-up based on ADC measurements is not ready to replace the current staging parameters or response assessment using the mRECIST criteria.

Acknowledgements We thank Mrs. N.S. Mostafavi for her advice on statistical analysis.

Funding This research received no specific Grant from any funding agency in the public, commercial, or non-profit sectors.

\section{Compliance with ethical standards}

Conflict of interest Heinz-Josef Klümpen is a member of the advisory board for Ipsen and Sirtex, and received an unrestricted research grant 
from Bayer (no grant numbers apply). R. Bart Takkenberg served as a speaker for Gore WL, Bayer, and Norgine. He is a member of the advisory board for Gilead and Norgine. He has received grants from the Netherlands Organisation for Health Research and Development (Zon$\mathrm{Mw}$ ) and the Netherlands Society for Gastroenterology (Gastrostart). Otto M. van Delden served as a consultant for Cook Medical. All other authors have declared no conflicts of interest. The study was designed and conducted by academic investigators.

Open Access This article is distributed under the terms of the Creative Commons Attribution 4.0 International License (http://creativeco mmons.org/licenses/by/4.0/), which permits unrestricted use, distribution, and reproduction in any medium, provided you give appropriate credit to the original author(s) and the source, provide a link to the Creative Commons license, and indicate if changes were made.

\section{References}

1. Park JW, Chen M, Colombo M, Roberts LR, Schwartz M, Chen PJ, et al. Global patterns of hepatocellular carcinoma management from diagnosis to death: the BRIDGE Study. Liver international : official journal of the International Association for the Study of the Liver. 2015;35(9):2155-66.

2. Galle PR, Forner A, Llovet JM, Mazzaferro V, Piscaglia F, Raoul J-L, et al. EASL Clinical Practice Guidelines: Management of hepatocellular carcinoma. Journal of hepatology. 2018;69(1):182-236.

3. Raoul JL, Sangro B, Forner A, Mazzaferro V, Piscaglia F, Bolondi $\mathrm{L}$, et al. Evolving strategies for the management of intermediatestage hepatocellular carcinoma: available evidence and expert opinion on the use of transarterial chemoembolization. Cancer treatment reviews. 2011;37(3):212-20.

4. Bruix J, Reig M, Sherman M. Evidence-Based Diagnosis, Staging, and Treatment of Patients With Hepatocellular Carcinoma. Gastroenterology. 2016;150(4):835-53.

5. Lencioni R, Llovet JM. Modified RECIST (mRECIST) assessment for hepatocellular carcinoma. Seminars in liver disease. 2010;30(1):52-60.

6. Calderaro J, Couchy G, Imbeaud S, Amaddeo G, Letouze E, Blanc JF, et al. Histological subtypes of hepatocellular carcinoma are related to gene mutations and molecular tumour classification. Journal of hepatology. 2017;67(4):727-38.

7. Malayeri AA, El Khouli RH, Zaheer A, Jacobs MA, CoronaVillalobos CP, Kamel IR, et al. Principles and applications of diffusion-weighted imaging in cancer detection, staging, and treatment follow-up. Radiographics: a review publication of the Radiological Society of North America, Inc. 2011;31(6):1773-91.

8. Gluskin JS, Chegai F, Monti S, Squillaci E, Mannelli L. Hepatocellular Carcinoma and Diffusion-Weighted MRI: Detection and Evaluation of Treatment Response. Journal of Cancer. 2016;7(11):1565-70.

9. Chen J, Wu M, Liu R, Li S, Gao R, Song B. Preoperative evaluation of the histological grade of hepatocellular carcinoma with diffusion-weighted imaging: a meta-analysis. PloS one. 2015;10(2): e0117661.

10. Shenoy-Bhangle A, Baliyan V, Kordbacheh H, Guimaraes AR, Kambadakone A. Diffusion weighted magnetic resonance imaging of liver: Principles, clinical applications and recent updates. World journal of hepatology. 2017;9(26):1081-91.

11. Suh YJ, Kim MJ, Choi JY, Park MS, Kim KW. Preoperative prediction of the microvascular invasion of hepatocellular carcinoma with diffusion-weighted imaging. Liver transplantation : official publication of the American Association for the Study of Liver
Diseases and the International Liver Transplantation Society. 2012;18(10):1171-8.

12. Kamel IR, Bluemke DA, Eng J, Liapi E, Messersmith W, Reyes DK, et al. The role of functional MR imaging in the assessment of tumor response after chemoembolization in patients with hepatocellular carcinoma. Journal of vascular and interventional radiology : JVIR. 2006;17(3):505-12.

13. Kamel IR, Bluemke DA, Ramsey D, Abusedera M, Torbenson $\mathrm{M}$, Eng J, et al. Role of diffusion-weighted imaging in estimating tumor necrosis after chemoembolization of hepatocellular carcinoma. AJR American journal of roentgenology. 2003;181(3):708-10.

14. Bonekamp S, Jolepalem P, Lazo M, Gulsun MA, Kiraly AP, Kamel IR. Hepatocellular carcinoma: response to TACE assessed with semiautomated volumetric and functional analysis of diffusion-weighted and contrast-enhanced MR imaging data. Radiology. 2011;260(3):752-61.

15. Vandecaveye V, Michielsen K, De Keyzer F, Laleman W, Komuta $\mathrm{M}$, Op de beeck K, et al. Chemoembolization for hepatocellular carcinoma: 1-month response determined with apparent diffusion coefficient is an independent predictor of outcome. Radiology. 2014;270(3):747-57.

16. Wu L, Xu P, Rao S, Yang L, Chen C, Liu H, et al. ADCtotal ratio and $\mathrm{D}$ ratio derived from intravoxel incoherent motion early after TACE are independent predictors for survival in hepatocellular carcinoma. Journal of magnetic resonance imaging : JMRI. 2017;46(3):820-30.

17. Guo Y, Yaghmai V, Salem R, Lewandowski RJ, Nikolaidis P, Larson $\mathrm{AC}$, et al. Imaging tumor response following liver-directed intra-arterial therapy. Abdominal imaging. 2013;38(6):1286-99.

18. Mannelli L, Kim S, Hajdu CH, Babb JS, Clark TW, Taouli B. Assessment of tumor necrosis of hepatocellular carcinoma after chemoembolization: diffusion-weighted and contrast-enhanced MRI with histopathologic correlation of the explanted liver. AJR American journal of roentgenology. 2009;193(4):1044-52.

19. Yuan Z, Li WT, Ye XD, Peng WJ, Xiao XS. Utility of diffusionweighted imaging to assess hepatocellular carcinoma viability following transarterial chemoembolization. Oncology letters. 2014;8(2):831-6.

20. Bonekamp S, Halappa VG, Geschwind JF, Li Z, Corona-Villalobos CP, Reyes D, et al. Unresectable hepatocellular carcinoma: MR imaging after intraarterial therapy. Part II. Response stratification using volumetric functional criteria after intraarterial therapy. Radiology. 2013;268(2):431-9.

21. Kokabi N, Camacho JC, Xing M, Edalat F, Mittal PK, Kim HS. Immediate post-doxorubicin drug-eluting beads chemoembolization Mr Apparent diffusion coefficient quantification predicts response in unresectable hepatocellular carcinoma: A pilot study. Journal of magnetic resonance imaging : JMRI. 2015;42(4):981-9.

22. Corona-Villalobos CP, Halappa VG, Bonekamp S, Eng J, Reyes $\mathrm{D}$, Cosgrove D, et al. Functional magnetic resonance imaging response of targeted tumor burden and its impact on survival in patients with hepatocellular carcinoma. Investigative radiology. 2015;50(4):283-9.

23. Dong S, Ye XD, Yuan Z, Xu LC, Xiao XS. Relationship of apparent diffusion coefficient to survival for patients with unresectable primary hepatocellular carcinoma after chemoembolization. European journal of radiology. 2012;81(3):472-7.

24. Lammer J, Malagari K, Vogl T, Pilleul F, Denys A, Watkinson A, et al. Prospective randomized study of doxorubicin-elutingbead embolization in the treatment of hepatocellular carcinoma: results of the PRECISION V study. Cardiovasc Intervent Radiol. 2010;33(1):41-52.

25. Evaluation of Sorafenib in Combination With Local Micro-therapy Guided by Gd-EOB-DTPA Enhanced MRI in Patients With Inoperable Hepatocellular Carcinoma [updated 5 December 2016. 
Available from:https://clinicaltrials.gov/ct2/show/study/NCT01 126645

26. Gillmore R, Stuart S, Kirkwood A, Hameeduddin A, Woodward $\mathrm{N}$, Burroughs AK, et al. EASL and mRECIST responses are independent prognostic factors for survival in hepatocellular cancer patients treated with transarterial embolization. Journal of hepatology. 2011;55(6):1309-16.

27. Kim BK, Kim SU, Kim MJ, Kim KA, Kim DY, Park JY, et al. Number of target lesions for EASL and modified RECIST to predict survivals in hepatocellular carcinoma treated with chemoembolization. Clinical cancer research : an official journal of the American Association for Cancer Research. 2013;19(6):1503-11.

28. Lencioni R. New data supporting modified RECIST (mRECIST) for Hepatocellular Carcinoma. Clinical cancer research : an official journal of the American Association for Cancer Research. 2013;19(6):1312-4.
29. Shim JH, Lee HC, Kim SO, Shin YM, Kim KM, Lim YS, et al. Which response criteria best help predict survival of patients with hepatocellular carcinoma following chemoembolization? A validation study of old and new models. Radiology. 2012;262(2):708-18.

30. Kamel IR, Liapi E, Reyes DK, Zahurak M, Bluemke DA, Geschwind JF. Unresectable hepatocellular carcinoma: serial early vascular and cellular changes after transarterial chemoembolization as detected with MR imaging. Radiology. 2009;250(2):466-73.

Publisher's Note Springer Nature remains neutral with regard to jurisdictional claims in published maps and institutional affiliations. 